U $m$ Google Book Search (GBS) ist in den letzten Wochen erbittert gestritten worden. Warum der plötzlich so massive Streit? GBS wurde in den letzten fünf Jahren eigentlich nur in einer engen Fachwelt der Informationsspezialisten diskutiert. Google macht seit etwa fünf Jahren Ernst mit dem Vorhaben, sämtliche Texte, die je in gedruckter Form erschienen sind, zu digitalisieren. Das, so sollte man meinen, wäre eigentlich der Generationenauftrag an die Bibliotheken. Die werden aber seit Jahren von ihren (zumeist öffentlichen) Trägern trotz aller politischen Verlautbarungen gleich zweifach im Stich gelassen: die Finanzmittel werden nicht bereitgestellt, und das Urheberrecht ist so verkrustet, dass es mehr als Verhinderung denn als Beförderung des Ziels großer digitaler Bibliotheken wirkt. Der weitaus größte Teil der Bestände der Bibliotheken ist in rechtsunsicheren Verhältnissen. Bibliothekare können und dürfen nicht.

Google hingegen kann und nimmt sich das Recht. Auf zwei Wegen: Einmal über das Verlagsprogramm. Google hat sich inzwischen mit weit über 20000 Verlagen geeinigt, die und nicht die Autoren selber - in der Regel die Rechteinhaber an den Werken sind. Google darf demnach in einem von dem jeweiligen Verlag zu bestimmenden Ausmaß Teile der Werke in GBS anzeigen. Bei dem anderen Weg, dem Bibliotheksprogramm, hat Google zunächst große US-amerikanische Bibliotheken und etwa 30 groBe private und öffentliche Bibliotheken aus aller Welt davon überzeugen können, dass es in ihrem ureigenen Interesse liege, wenn Google ihre gesamten Bestände digitalisiert.

Bei etwa 10 Mio. Büchern/Volltexten ist Google derzeit schon angelangt. $\mathrm{Ob}$ das alles rechtens war, was von Google digitalisiert wurde, z.B. vergriffene oder verwaiste Werke (letztere, die noch urheberrechtsgeschützt sind, für die die Rechteinhaber aber nicht ausgemacht werden

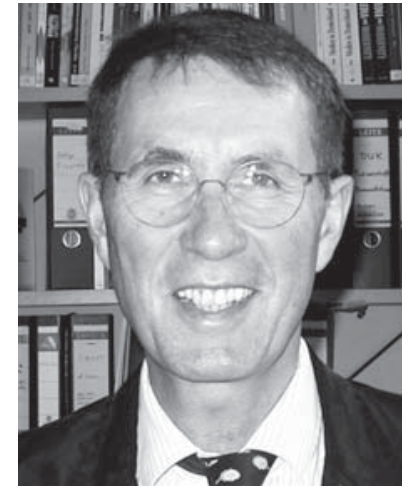

Rainer Kuhlen

\section{Buchdigitalisierung durch Google}

können), hat Google kaum interessiert. Wer Rechte nicht mehr wahrnimmt, soll, so vermutlich Google, sie auch nicht mehr exklusiv beanspruchen dürfen.

Zu der großen Empörung hierzulande kam es auch nicht, als sich vor über einem Jahr ein kleiner USamerikanischer Autoren- und ein ebensolcher Verlegerverband daran machte, Google wegen Rechteverletzungen zu verklagen. Zu einer Entscheidung kam es nicht. Wie üblich, wenn Milliarden beteiligt sind, wurde ein Vergleich ausgehandelt. Dieser Vergleich würde Google berechtigen, auch urheberrechtsgeschützte Werke aus aller Welt, die in Bibliotheken in den USA sind, in GBS einzuspeisen. Dies hat nicht nur in Deutschland große Empörung hervorgerufen. Wie könne es denn sein, dass über einen inner-amerikanischen Vergleich weltweite Rechte an Werken, über die Autoren oder Verlage exklusive Rechte besitzen, Google zugebilligt werden. In Deutschland hat sich das Justizministerium (BMJ) am radikalsten gegen diesen Vergleich gewandt und das New Yorker Gericht gebeten, diesen gänzlich abzulehnen oder zumindest deutsche geschützte Werke auszunehmen.

Es geht bei dieser Debatte weder um das Infragestellen von oder um das Beharren auf Prinzipien wie geistiges Eigentum. Es geht schlicht um Märkte und Interessen. Wie auch der Vergleich entschieden wird - ich wage die Prognose, dass er in der jetzigen Form nicht angenommen werden wird -, Google wird seine Digitalisierungspolitik fortsetzen. Daher ist es durchaus im Interesse der Autoren, wenn mit Google direkt geeignete Modelle ausgehandelt werden. Für die Wissenschaft hat z.B. das Aktionsbündnis Urheberrecht für Bildung und Wissenschaft Gespräche mit Google in Kalifornien begonnen. Dabei werden gegen die Digitalisierung der wissenschaftlichen Werke keine Einwände erhoben. Ob Autoren außerhalb der Wissenschaft auch nicht besser fahren würden, wenn für sie passende Bedingungen direkt ausgehandelt würden, sei dahingestellt. Im Grunde stehen jedoch nur die Interessen der Verlage auf dem Spiel, nicht die der Autoren, deren Primärinteresse es ist, so weit wie möglich sichtbar zu sein. Kommerzielle Vorteile haben Autoren bei einer Verweigerung gegenüber GBS nicht.

Auch die Wirtschaft insgesamt zöge Nutzen aus einer freien Verfügbarkeit wissenschaftlicher Werke, die derzeit nicht mehr auf dem Markt erhältlich sind. Den Interessen von Bildung und Wissenschaft, aber auch der generellen Wirtschaft, außerhalb der Publikationsindustrie, wird allerdings durch die strikte Ablehnungspoltik des Bundes kaum Rechnung getragen. Aber man kann dem Ganzen auch eine hoffnungsfroh positive Seite abgewinnen. Vielleicht sehen sich die öffentlichen Träger nun doch veranlasst, in die sich nur lahm entwickelnden öffentlichen digitalen Bibliotheken zu investieren, sowohl in finanzieller als auch in rechtlicher Hinsicht. Google ermöglicht sozusagen, über Umwege das Ziel der freien Verfügbarkeit von Wissen und Information zu erreichen.

Rainer Kuhlen ist Professor an der Universität Konstanz - Fachbereich Informatik und Informationswissenschaft rainer.kuhlen@uni-konstanz.de Wirtschaftsdienst $2009 \bullet 9$ 